\title{
Development and Psychometric Properties of Cognitive Appraisals of Health Situation Scale In Persons with Spinal Cord Injury Due To Traumatic Incidents In Iran
}

\author{
Maryam Shabany \\ Tehran University of Medical Sciences
}

Seyed Mohammad Ghodsi

Tehran University of Medical Sciences

Roya Habibi Arejan

Tehran University of Medical Sciences

Reza Tabrizi

Tehran University of Medical Sciences

Parastoo Saberi

Tehran University of Medical Sciences

Vali Baigi

Tehran University of Medical Sciences

Zahra Ghodsi

Tehran University of Medical Sciences

Fatemeh Rakhshani

Tehran University of Medical Sciences

Vafa Rahimi-Movaghar ( $\nabla_{\text {v_rahimi@sina.tums.ac.ir) }}$

Sina Trauma and Surgery Research Center

Alex R. Vaccaro

Thomas Jefferson University

Original research

Keywords: Validity, Reliability, Cognitive appraisal, Spinal cord injury

Posted Date: September 7th, 2021

DOl: https://doi.org/10.21203/rs.3.rs-864713/v1

License: (c) (i) This work is licensed under a Creative Commons Attribution 4.0 International License. Read Full License 
Page $2 / 22$ 


\section{Abstract}

Study Design: Development and psychometrics study

Objective: To evaluate the reliability and validity of a new version of cognitive appraisals of health situation scale in Traumatic Spinal Cord Injury (CAHSSTSCI) in the Persian language for persons with spinal cord injury (SCl).

Setting: The persons were selected from National Spinal Cord Injury Registry of Iran (NSCIR-IR) and Brain and Spinal Cord Injury Research center (BASIR).

Method: This was a mixed sequential exploratory study that performed in two phases. In the qualitative phase, a systematic scoping review and 12 interviews with the participants were done. Finally, items were generated. In the quantitative phase, to evaluate the validity; face, content, construct and convergent validity were assessed. To evaluate construct validity, a cross-sectional study was conducted on 305 persons with $\mathrm{TSCl}$, and also the reliability was examined by internal consistency and stability assessment. All quantitative data analyses were conducted using SPSS 20 software.

Results: The content validity and reliability were indicated by Scale's Content Validity Ratio (S-CVR) $=0.73$ and Scale's Content Validity Index $(\mathrm{S}-\mathrm{CVI})=0.86$, Cronbach's $\mathrm{a}=0.9$ and the Test re-test reliability using intra-class correlations were $(I C C)=0.97$ to 0.98 . Exploratory factor analysis determined eight factors which showed more than $52 \%$ of the variance. CAHSSTSCI had a significant and strong correlation with Appraisals of DisAbility Primary and Secondary Scale (ADAPSS) $(r=0.475, P<0.001)$.

Conclusion: Results showed the 36 items CAHSSTSCI tool had an acceptable validity and reliability in Iran and it can help health care providers or even administrators to improve the quality of the rehabilitation services and quality of life through design training programs for SCl people due to traumatic incidents.

\section{Introduction}

Some people experience traumatic incidents at some time in their lives. Traumatic incidents such as motor vehicle and transportation accidents, falls, violence, sports, or earthquakes may lead to spinal cord injury $(\mathrm{SCl})(1,2)$. Most of the affected persons with $\mathrm{SCl}$ are due to trauma including traffic accidents and falls. In many countries, traumatic SCI (TSCl) has not been studied epidemiologically(3). Since TSCl is a sudden event, it causes severe problems in various aspects of a person's life including physical, psychological, social, and economic $(4,5)$.

Disability following a traumatic event can create various challenges in a person's life $(6,7)$. Responses to this debilitating condition can be varied and it may depend on the cognitive appraisal of the new situation(6). Indeed Cognitive appraisal or simply "appraisal" refers to the way persons evaluate the 
relationships with their environment, and more to the importance of these relationships to their health and wellbeing(8).

If a situation threatens a person's health and the person considers the event as a threat, loss, or challenge, the person begins to cope with it which is called initial appraisal. In addition evaluation of their resources and the options for coping is secondary appraisal. Both primary and secondary appraisals run simultaneously and are interrelated(8).

Measurement of cognitive appraisal is considered a relatively new field of study, since most evaluation scales have only been developed over the past two decades $(8,9)$. It is necessary to make a scale for identifying cognitive appraisals of people exposed to various types of potentially stress-related events(10). There is little research on the cognitive appraisal of SCI following road accidents and traumatic incident. Kennedy et al. has conducted research in this field and found that cognitive appraisal can be a strong predictor of stress and anxiety in people with spinal cord injury(11). This inappropriate behavioral response may negatively affect the well-being and quality of life of these people (10).

There are various tools for measuring cognitive appraisals, although some of them only measure initial evaluation and others secondary measurements, and few tools measure both(12). Furthermore, the translation of the tool alone is not enough, and the underlying cultural differences must be fully respected(13). An instrument designed in one special country can only reflect the language and culture of that country, and if it is used in another country, many problems can arise due to the lack of content similarity(14). To achieve acceptable validity and reliability, the tool should be based on the community culture in the study. Content extraction through the participation of respondents in a structured and scientific way in different countries is a scientific necessity that maximizes the cultural adaptation of the instrument of measurement(13). In fact, a tool that specifically assesses cognitive appraisal in relation to the condition caused by the $\mathrm{SCl}$ and considers different physical, psychological and social aspects of this period in Iran has not been designed. Therefore, the present study aims to design and validate a cognitive appraisal questionnaire for Iranian people with SCl following traumatic incidents.

\section{Method}

A mixed sequential exploratory study was used to develop a valid and reliable questionnaire to determine Cognitive Appraisals of Health Situation Scale in TSCI (CAHSSTSCI).

The present study was conducted in two qualitative and quantitative phases for tool development(15).

\section{Qualitative phase}

\section{- Study design in the qualitative phase}


This phase is related to development of the tool. Indeed, an inductive-deductive approach was conducted to generate appropriate initial items. First, a systematic scoping literature review was performed. Then a qualitative study with the content analysis approach was done to explain the concept of cognitive appraisal from the persons with $\mathrm{SCl}$ following an accident or traumatic incident (16). The items were extracted from both studies.

\section{- The review process}

A systematic scoping literature review was performed using key words and search strategy was designed by a specialist in library sciences and medical research. Some of key words derived from MeSH, Emtree terms, and free text words included "Spinal Cord Injuries", "Trauma", "cognitive", "Appraisal", "health status" through databases, PubMed, Web of Science, Scopus, and Embase from 1990 to 16 May 2020, without any language limitation.

\section{- The participants}

Participants were selected using a purposive sampling (identification and selection of information-rich cases related to the phenomenon) (17) from NSCIR from Trauma research center and Brain and Spinal Cord injury Research (BASIR) center in Tehran. Participants were people with $\mathrm{TSCl}$ who all agreed to participate in this study. All participants had TSCl for at least 6 months.

Maximum variation in participant demographics was prioritized during the sampling (gender, different socio-economic levels, and different clinical situation)(18).

\section{- The process of interview and data collection}

After explaining the purpose of the study to the participants, the researcher reassured them about confidentiality of their personal information. Ultimately, written consent for data collection was obtained. A deep face to face, semi-structured individual interview with open-ended questions was conducted. In the interview, participants were encouraged to share their experiences about the $\mathrm{TSCl}$ and related health problems. Some questions are in Appendix1, part A.

The interview was continued until data saturation when no new code of data was added to the study. In general, 12 people participated from July 2020 to March 2021 in this study. The interviews were done by the first author who has qualitative research experience and experience working with people with TSCl. Also, field notes were made during and after the interview. The average duration of interviews was 60 to 90 minutes.

\section{- Data analysis}


Data analysis was performed with qualitative conventional content analysis approach which was designed by Graneheim and Lundman (19). MAXQDA v.2018 was used for data management.

\section{- Rigor}

The codded data were assessed by people with SCl, peers, and experts and their suggestions such as correct sentences clearly and understandably were considered. Credibility, transferability, conformability, and dependability of the qualitative data were evaluated by the method proposed by Lincoln and Guba's criteria (20).

\section{Quantitative phase: psychometric properties of CAHSSTSCI}

The psychometric properties of the questionnaire were assessed as follows:

Quantitative and qualitative face validity, quantitative and qualitative content validity, constructs validity, and the reliability of the tool.

\section{- Face validity assessment}

As a pre-test, it was performed both qualitatively and quantitatively. To determine the qualitative face validity, 10 people with $\mathrm{SCl}$ caused by traumatic accidents and 15 health care providers in this field were interviewed by voice and video online. Potential problems arose regarding the understanding of the items or words, the existence of ambiguity, and misinterpretations of items. The meanings of the sentences were examined. At this stage, some items were corrected. The following formula was used for calculating face validity:

Item impact score $=$ Frequency of response $($ percentage $) \times$ Importance of item (21).

Content Validity: Content validation is usually used to examine the components of a measuring instrument. In this stage, the preliminary questionnaire was evaluated by 15 health care providers who previously published articles about coping, cognitive appraisals and related subjects: 4 psychologists, 1 psychiatrists, $2 \mathrm{PhD}$ in health education, $3 \mathrm{PhD}$ in disaster health, $3 \mathrm{PhD}$ in nursing who were specialist in long term care and psychology nursing, and 2 Neuro-trauma epidemiologists. In the present research, the opinion of the experts was used to provide quantitative credit (content validity ratio (CVR) and content validity index (CVI) calculation) and qualitative validity (grammar, use of proper words, etc.) of the questionnaire.

To calculate the CVR score, the items were rated based on the 3 options: "necessary", "useful but not necessary", and "not necessary." Comments or suggestions about the content of the items were also collected. The content validity ratio was evaluated based on the 2014 Lawshe(22) table and the 
minimum acceptable score of CVR per 15 experts was 0.6. Finally, out of the items selected for CVR, 14 were removed and 36 were left, which were examined for content validity index (CVI) in the following table.

Furthermore, the CVI was assessed by 15 experts who scored items of the questionnaire based on their "simplicity", "relevance" and "clarity" using the 4-level Likert scale (scores 0 to 3 for "not at all "to "completely") based on Waltz \& Bausell's content validity index(23). CVI was calculated with the following formula:

(CVI= (Number of raters choosing points 3 and $4 /$ Total number of raters))

Items with a CVI more than 0.79 , between 0.70 and 0.79 , and less than 0.70 were considered suitable, needing modification, and unacceptable, in order (23). The scale's CVR (S-CVR) and the scale's CVI (S$\mathrm{CVI}$ ) were acquired through calculating of mean of items' CVR and CVI.

Comprehensiveness of the tool: The ability of the tool to cover all areas related to the subject under study constitutes the comprehensiveness of the tool. Four options were used: "completely comprehensive", "comprehensive", "somewhat comprehensive", "incomplete". To calculate this index, the total number of "completely comprehensive" specialists who have recognized the comprehensiveness of the tool as comprehensive and completely comprehensive was divided by the total number of specialists. If the comprehensiveness score of the confirmation questionnaire / overall comprehensiveness of the instrument was equal or greater than 0.7 .

\section{Construct validity}

In this research, construct validity of CAHSSTSCI was evaluated through exploratory factor analysis (EFA) using principal component analysis (PCA) (24).

\section{- Convergent validity}

To evaluate the convergent validity, the correlation between new scale (CAHSSTSCI) and the Appraisals of DisAbility Primary and Secondary Scale (Kennedy et al.)(10) was assessed.

\section{- Sampling}

Plichta et al. cited that the required number of participants for EFA is between 3 and 10 persons per item (25). In this study, we tried to select the maximum number of samples, about 8 people were considered for 36 question, so a total of 305 persons with TSCI were selected of NSCIR-IR and brain and SCI center (BASIR) affiliated Tehran University of Medical Sciences (TUMS) in this study. For the sampling, the researcher used of respondents who were available (convenience method). The inclusion criteria were: 
persons with $\mathrm{SCl}$ due to an accident or traumatic incident that occurred more than 6 months ago, willingness to participate, knowing Persian language, no amputation in the limbs, no coma in the last six months, and no severe mental problems.

\section{- Data analysis}

Two criteria of Kaiser-Meyer-Alekin (KMO) and Bartlett Sphericity Test were used to confirm if there is enough correlation between the variables for EFA. The sample size sufficiency for EFA can be calculated $\mathrm{KMO}$ index is more than 0.8 and the p-value of the Bartlett Sphericity test is less than 0.05(26). After that, the items were tested about propriety to enter factor analysis by calculating commonalities. And then, items with commonalities of more than 0.4 were selected for the analysis. According to the Kaiser (1960) criterion that is the acceptance of factors having an Eigen value of more than one and also drawing the Scree plot, the factors of the tool were extracted. However, for more accuracy, parallel analysis was used for determining the exact number of factors.(27).

\section{- Reliability}

For the reliability the internal consistency assessment and Stability assessment were done:

\section{- The internal consistency assessment}

The internal consistency of CAHSTSCI was calculated by the Cronbach's alpha coefficient, and the values higher than 0.7 were considered acceptable (28). To assess internal consistency, 58 eligible persons with $\mathrm{SCl}$ filled the questionnaire.

\section{- Stability assessment}

Stability of CAHSTSCI was assessed through the test-retest method. This questionnaire was completed twice within an interval of 10 days by 58 eligible persons with $\mathrm{SCl}$. For the scores of two tests, Pearson correlation and intra-class correlation coefficients (ICC) were used.

The Pearson correlation coefficient more than 0.7 [23] and ICC higher than 0.4 were considered as the acceptable levels for stability [30]. The SPSS version 22 was used to calculate all statistical analyses.

A summary of steps in the current method were presented in Appendix 1 part B.

\section{Results}

The Findings are reported in two parts: 


\section{1) Designing of the CAHSSTSCl; and 2) Assessing the psychometric properties of the CAHSSTSCI.}

\section{1) Designing of the CAHSSTSCI (Qualitative phase)}

According to the extracted concept and definition, the items of the questionnaire were made. The extracted items from the qualitative part and the literature review made the primary pool of 115 items (59 = qualitative part, $56=$ literature review). The overlapping items were merged and the unrelated items were removed and the number of items was reduced to 52 items at this stage. A questionnaire was designed with the help of these items. Finally, this 52-item questionnaire was designed and evaluated. The items were classified in 11 categories in qualitative phase (Appendix 1 part B and C).

\section{2) Assessing the psychometric properties of the CAHSSTSCI (Quantitative phase)}

In this phase, four items were omitted for duplicate concept, ambiguity, or generality. Indeed, for quantitative face validity assessment, the importance of each item was computed and the items with impact score of more than 1.5 were retained. In this stage, 48 phrases had a score of more than 1.5.

\section{Content validity assessment}

In this part, 12 items were deleted as they didn't obtain acceptable CVI and CVR level. In general, content validity of CAHSSTSCI was assessed by S-CVR and S-CVI, 0.73 and 0.86 , respectively. Then, the questionnaire with 36 items entered the stage of construct validity assessment. Furthermore, the comprehensiveness of the instrument in this study was calculated to be $86.66 \%$.

\section{Construct validity assessment}

The EFA using PCA method was performed to assess the construct validity of CAHSSTSCI. Demographic and clinical variables of the participants for 305 persons with TSCl are shown in Table 1. For sampling adequacy Kaiser-Meyer-Olkin Measure (KMO index) was calculated and it was 0.734 , and also the Bartlett Sphericity test showed the correlation matrix to be 5442.545 with $\mathrm{P}<0.0001$. Both of the tests showed sufficient correlation between variables for EFA.

Scree plot and parallel analysis suggested 8 factors to predict the number of factors. The parallel analysis and scree plot was used as the default for factor analysis. Eight factors that explained $61.60 \%$ of cumulative variance of CAHSSTSCI were identified using the minimum eigenvalues of 1.31. Furthermore, Quartimax rotation and considering the factor loading of at least 0.4 , the items forming each factor were identified (Table 2). 
Each factor was named and it had a number of items. These factors were categorized and named in Table 2, respectively. The "Perception threat due to $\mathrm{SCl}=1$ " with 6 items explaining $14.19 \%$ of variance, "Resilience=2" with 7 items explaining $8.18 \%$ of variance, "Losing=3" with 4 items explaining $7.76 \%$ of variance, " Compatibility=4" explaining with 7 items $7.57 \%$ of variance, "Post-traumatic growth=5" with 4 items explaining $7.32 \%$ of variance, "Vague understanding $=6$ " with 3 items of being explaining $5.69 \%$ of variance, "Disbelief and anger $=7$ " with 3 items explaining $5.66 \%$ of variance, "Improper communication=8" with the environment with 2 items explaining $5.21 \%$ of variance.

\section{Convergent Validity}

Seventy persons with TSCI completed two questionnaires of CAHSSTSCI and ADAPSS. The results showed that there was a positive and significant correlation between the two $(p=0.00,0.475)$. This correlation indicates that the two scales measure common structures but are not exactly the same. Thus, the convergent validity was confirmed.

\section{Reliability}

Internal consistency and stability were assessed for CAHSTSCI. Internal consistency of CAHSTSCI was demonstrated by Cronbach a at 0.9 for whole instrument. Correlation coefficient and ICC of the whole questionnaire were reported 0.980 and 0.973 , respectively. Table 4 showed the results of questionnaire's reliability assessment. After confirming validity and reliability of the CAHSSTSCI, the questionnaire was finalized.

\section{Scoring procedure in CAHSSTSCI}

CAHSTSSCI was scored by a rating scale from 1 to 5 . The range of scores for the whole questionnaire and its subscales are presented in Table 4. The total score of the CAHSSTSCI and its subscales are calculated and presented as percentages. The range of scores is from $36(0 \%)$ to $180(100 \%)$.

Higher scores show that people with SCl have a good cognitive appraisal of health status and tend to cope with health problems. Subscales of "resilience" from 7 to 35, "compatibility" from 6 to 30 , and "posttraumatic growth" from 5 to 25 are related to coping and a positive cognitive appraisal of their health situation. Therefore, individuals with TSCl that acquired higher scores in these factors had positive cognitive appraisal. On the other hand, "perception threat due to SCl" from 6 to 30, "losing" from 4 to 20, "vague understanding of being" from 3 to 15, "disbelief and anger" from 3 to 15, and "improper communication with environment" from 2 to 10 were related with negative appraisal. Individuals with TSCl that acquired higher scores in these factors had negative cognitive appraisal. (Appendix 1, part D)

\section{Discussion}


CAHSSTSCI is a tool to assess cognitive appraisal of health in TSCl due to road traffic accidents, natural disaster, or other traumatic incidents in Iranian people. This valid and reliable tool is able to measure positive and negative appraisal of a person's health situation. It assesses how individuals with TSCl interpret their health situation (primary appraisal) and how they cope with the stressful event (secondary appraisal) following physical trauma. Therefore, the result of this questionnaire may help health care managers and planners to improve the quality of physical and psychological rehabilitation care or quality of life in TSCI people.

Roger Carpenter in a review article stated there are limited tools for assessing cognitive appraisal and a demand exists for making valid and reliable appraisal instruments for individuals experiencing stress due health problems(29). However, Dean and Kennedy studied the validity and reliability of Appraisals of DisAbility Primary and Secondary (ADAPSS) in spinal cord injury(10). These studies were similar to the present study.

However, our study is related to health problems caused by SCI. This means that both immobility and the side effect of immobility have also been considered. Also, different types of cervical and lumbar spinal cord injuries and wheelchair dependent and non-wheelchair users can complete this questionnaire.

Furthermore, varies dimensions (categories) for cognitive appraisal of a Peron's health situation emerged from the qualitative part and the literature review. Furthermore, after the EFA and parallel analysis, eight factors were indicated. Perception threat due to SCl, Resilience, Losing, Compatibility, Post-traumatic growth, Vague understanding of being, Disbelief and anger, and Improper communication with environment. Four dimensions of the present tool were similar to ADAPSS which was developed by Dean and Kennedy(10). The ADAPSS has a 6-factor construction with the following subscales: fearful despondency, overwhelming disbelief, determined resolve, growth and resilience, negative perceptions of disability, and personal agency (10).

In the present study, "Resilience" was composed of 7 items and explains the highest variance and predictability for better cognitive appraisal of health after SCI. "Compatibility" and "Perception threat due to SCl" were second regarding their predictability for cognitive appraisal of health in TSCI. The third factor was "post traumatic growth" with 5 items. The 3 factors of "resilience", "compatibility", and "post traumatic growth" were predictive of a better cognitive appraisal of the health situation after TSCI. These factors were a positive appraisal such as the factor of "challenge" response to a stressful event in the study by Muayyad Ahmad (30). However, these factors were somewhat fit with "determined resolve" and "growth and resilience" in the study of Dean and Kennedy(10).

On the other hand, "Perception threat due to SCl", "Losing", "Vague understanding of being", "Disbelief and anger", and "Improper communication with environment" respectively explain the variance rate for negative cognitive appraisal of health in TSCI. These factors were similar to fearful despondency, overwhelming disbelief, and negative perceptions of disability [9]. Indeed the present factors were similar to harm /loss and threat factors in Ahmad's study (31). 
In the present study, spirituality and environment were considered in the items which were not in previous studies. Taylor Newton and Daniel N. Mclntosh in their study showed that specific religious beliefs directly influenced cognitive appraisals(32). This result showed the relationship between spirituality and cognitive appraisals. In another study by Duggan et al, cited that the environment can be a stressor for women with $\mathrm{SCl}$ and it may have a negative impact on cognitive appraisal(33).

Internal consistency and stability of CAHSTSCI suggest high reliability of the questionnaire. The study defined the concept of cognitive appraisal of the health situation in $\mathrm{TSCl}$ as it can be measured by CAHSTSCl containing 8 subscales that predict $52 \%$ of variance.

CAHSTSCl showed gaps in assessment of cognitive appraisal in the health care system of Iran. There is no special limitation for this study.

\section{Conclusion}

CAHSSTSCI is a valid and reliable tool with 36 items to assess cognitive appraisal of health in TSCl by a five level Likert scale. It consists of 8 subscales including:

"resilience", "compatibility", and "post-traumatic growth" that were related to coping and a positive cognitive appraisal and "perception threat due to SCl", "losing", "vague understanding of being", "disbelief and anger", and "improper communication with environment" that were related to negative appraisal. This questionnaire can help health care providers and planners to design training programs according to cognitive appraisal of health problems after SCl. Furthermore, they can improve post-event psychological reactions, adjustment and resilience, the rehabilitation services, and ultimately improve the quality of life for disabling people after traumatic incidents.

\section{Declarations}

\section{Acknowledgements}

We would like to acknowledge Dr. Sally Kaiser for sending us helpful information about ADAPS scale.

\section{Author contributions}

VRM, MGh, MSh and VB contributed to designing and writing the method. In addition, FR, RH, VRM, ZGH helped to design and corrected questionnaire several time. PS and MSH were done data gathering. RT and $\mathrm{MSH}$ analyzed and interpreted the results. All authors wrote the article and help to critically review.

\section{Funding}


This work was funded by Sina Trauma and Surgery Research Center, Tehran University of Medical Sciences [grant number is 98-02-38-43250].

\section{Availability of data and materials}

The datasets analyzed during the current study are available from the corresponding author on reasonable request, with Ethics Committee approval.

\section{Compliance with ethical standards}

\section{Statement of Ethics}

The Ethics Committee of Sina Trauma and Surgery Research Center, Tehran University of Medical Sciences, approved the study, and the reference number is98-02-38-374.

We certify that all applicable institutional and governmental regulations were followed during the course of this research.

\section{Conflicts of interest}

The authors declare that they have no competing interests except Alexander R. Vaccaro (Appendix 2).

\section{References}

1. Wyndaele M, Wyndaele J-J. Incidence, prevalence and epidemiology of spinal cord injury: what learns a worldwide literature survey? Spinal cord. 2006;44(9):523-9.

2. Groves C, Poudel M, Baniya M, Rana C, House D. Descriptive study of earthquake-related spinal cord injury in Nepal. Spinal Cord. 2017;55(7):705-10.

3. Jazayeri SB, Beygi S, Shokraneh F, Hagen EM, Rahimi-Movaghar V. Incidence of traumatic spinal cord injury worldwide: a systematic review. European spine journal. 2015;24(5):905-18.

4. Noreau L, Proulx P, Gagnon L, Drolet M, Laramée M-T. Secondary impairments after spinal cord injury: a population-based study. American journal of physical medicine rehabilitation. 2000;79(6):526-35.

5. Mahmoodi N, Ai J, Ebrahimi-Barough S, Hassannejad Z, Hasanzadeh E, Basiri A, et al. Microtubule stabilizer epothilone $B$ as a motor neuron differentiation agent for human endometrial stem cells. Cell Biol Int. 2020;44(5):1168-83.

6. Woolrich RA, Kennedy P, Tasiemski T. A preliminary psychometric evaluation of the Hospital Anxiety and Depression Scale (HADS) in 963 people livingwith a spinal cord injury. Psychology Health 
Medicine. 2006;11(1):80-90.

7. Kyutoku Y, Tada R, Umeyama T, Harada K, Kikuchi S, Watanabe E, et al. Cognitive and psychological reactions of the general population three months after the 2011 Tohoku earthquake and tsunami. PloS one. 2012;7(2):e31014.

8. Lazarus RS, DeLongis A, Folkman S, Gruen R. Stress and adaptational outcomes: The problem of confounded measures. 1985.

9. King KR. Why is discrimination stressful? The mediating role of cognitive appraisal. Cultural Diversity Ethnic Minority Psychology. 2005;11(3):202.

10. Dean R, Kennedy P. Measuring appraisals following acquired spinal cord injury: A preliminary psychometric analysis of the appraisals of disability. Rehabilitation psychology. 2009;54(2):222.

11. Marshall RE. Measuring appraisals following acquired spinal cord injury: A psychometric analysis of the primary and secondary appraisals of disability scale. (PSADS): Oxford University; 2008.

12. Kessler TA. The cognitive appraisal of health scale: Development and psychometric evaluation. Res Nurs Health. 1998;21(1):73-82.

13. Sperber AD. Translation and validation of study instruments for cross-cultural research. Gastroenterology. 2004;126:124-S8.

14. Humeniuk R, Ali R, Babor TF, Farrell M, Formigoni ML, Jittiwutikarn J, et al. Validation of the alcohol, smoking and substance involvement screening test (ASSIST). Addiction. 2008;103(6):1039-47.

15. Rodgers BL, Knafl KA. Concept development in nursing: Foundation, techniques and application. 2nd ed. Philadelphia: W.B. Saunders; 2000. pp. 129e161.

16. Creswell JW, Creswell JD. Research design: Qualitative, quantitative, and mixed methods approaches. Sage publications; 2017.

17. Palinkas LA, Horwitz SM, Green CA, Wisdom JP, Duan N, Hoagwood K. Purposeful sampling for qualitative data collection and analysis in mixed method implementation research. Administration policy in mental health mental health services research. 2015;42(5):533-44.

18. Benoot C, Hannes K, Bilsen J. The use of purposeful sampling in a qualitative evidence synthesis: $A$ worked example on sexual adjustment to a cancer trajectory. BMC medical research methodology. 2016;16(1):1-12.

19. Lindgren B-M, Lundman B, Graneheim UH. Abstraction and interpretation during the qualitative content analysis process. International Journal of Nursing Studies. 2020:103632.

20. Guba EG, Lincoln YS. Guidelines and checklist for constructivist (aka fourth generation) evaluation. 2001.

21. Hajizadeh E, Asghari M. Statistical methods and analyses in health and biosciences a research methodological approach. Tehran: Jahade Daneshgahi Publications. 2011;395.

22. Ayre C, Scally AJ. Critical values for Lawshe's content validity ratio: revisiting the original methods of calculation. Measurement evaluation in counseling development. 2014;47(1):79-86. 
23. Waltz CF, Strickland OL, Lenz ER. Measurement in nursing and health research. Springer publishing company; 2010.

24. Kawulich BB. editor Participant observation as a data collection method. Forum qualitative sozialforschung/forum: Qualitative social research; 2005.

25. Munro BH. Statistical methods for health care research. lippincott williams \& wilkins; 2005.

26. Plichta SB, Kelvin EA, Munro BH. Munro's statistical methods for health care research. Wolters Kluwer Health/Lippincott Williams \& Wilkins; 2013.

27. Watkins MW. Determining parallel analysis criteria. Journal of modern applied statistical methods. 2006;5(2):344-6.

28. Alwin DF. How good is survey measurement? Assessing the reliability and validity of survey measures. Handbook of survey research. 2010;2:405-34.

29. Carpenter R. A review of instruments on cognitive appraisal of stress. Arch Psychiatr Nurs. 2016;30(2):271-9.

30. Ahmad MM. Psychometric evaluation of the Cognitive Appraisal of Health Scale with patients with prostate cancer. Journal of advanced nursing. 2005;49(1):78-86.

31. Ahmad MM. Validation of the cognitive appraisal health scale with Jordanian patients. Nurs Health Sci. 2010;12(1):74-9.

32. Newton AT, Mclntosh DN. Specific religious beliefs in a cognitive appraisal model of stress and coping. International Journal for the Psychology of Religion. 2010;20(1):39-58.

33. Duggan $\mathrm{CH}$, Albright $\mathrm{KJ}$, Lequerica A. Using the ICF to code and analyse women's disability narratives. Disabil Rehabil. 2008;30(12-13):978-90.

\section{Tables}

Table 1

Demographic and clinical variables of the participants 


\begin{tabular}{|c|c|c|}
\hline \multicolumn{2}{|l|}{ Variables } & \multirow{2}{*}{$\begin{array}{l}\mathrm{N}(\%) \\
91(29.8)\end{array}$} \\
\hline Age & $\geq 30$ & \\
\hline \multicolumn{2}{|c|}{$31-45$} & $159(52.1)$ \\
\hline \multicolumn{2}{|l|}{$46-60$} & $47(15.4)$ \\
\hline \multicolumn{2}{|l|}{$61 \leq$} & $8(2.6)$ \\
\hline \multirow[t]{2}{*}{ Sex } & Man & $259(84.91)$ \\
\hline & Woman & $46(15.09)$ \\
\hline \multicolumn{3}{|c|}{ Educational level } \\
\hline \multicolumn{2}{|c|}{ illiterate } & $1(0.3)$ \\
\hline \multicolumn{2}{|c|}{ Primary school } & $58(19)$ \\
\hline \multicolumn{2}{|c|}{ Secondary school } & $74(24.3)$ \\
\hline \multicolumn{2}{|c|}{ High school } & $151(49.5)$ \\
\hline \multicolumn{2}{|c|}{ Bachelor } & $16(5.2)$ \\
\hline \multicolumn{2}{|c|}{ Master of science } & $3(1)$ \\
\hline \multicolumn{2}{|c|}{ Doctor/PhD } & $2(0.7)$ \\
\hline \multicolumn{3}{|c|}{ Marital status } \\
\hline \multicolumn{2}{|c|}{ Single } & $5(1.6)$ \\
\hline \multicolumn{2}{|c|}{ Married } & $106(34.8)$ \\
\hline \multicolumn{2}{|c|}{ Divorced } & 194(63.6) \\
\hline \multirow[t]{3}{*}{ Location } & Capital (Tehran) & $64(20.98)$ \\
\hline & Other city & $235(77.04)$ \\
\hline & Village & $6(1.96)$ \\
\hline \multicolumn{3}{|c|}{ Cause of injury } \\
\hline \multicolumn{2}{|c|}{ Natural disaster } & $4(1.3)$ \\
\hline \multicolumn{2}{|c|}{ Road traffic accident } & $166(54.4)$ \\
\hline \multicolumn{2}{|c|}{ Falling } & $112(36.7)$ \\
\hline \multicolumn{2}{|c|}{ Hit of the bullet } & $2(0.7)$ \\
\hline \multicolumn{2}{|c|}{ Other transport } & $12(3.9)$ \\
\hline \multicolumn{3}{|c|}{ Incident } \\
\hline \multicolumn{2}{|c|}{ Blunt Trauma } & $9(3)$ \\
\hline
\end{tabular}




\begin{tabular}{|c|c|}
\hline \multicolumn{2}{|l|}{ Economic status } \\
\hline \multirow[b]{2}{*}{ Middle } & 216(70.8) \\
\hline & $89(29.2)$ \\
\hline \multicolumn{2}{|l|}{ Duration of SCl (month) } \\
\hline $12 \geq$ & $34(11.1)$ \\
\hline $13-60$ & $178(58.4)$ \\
\hline $61-120$ & $64(21)$ \\
\hline $121 \leq$ & $29(9.5)$ \\
\hline Level of SCI Paraplegia & 265(86.9) \\
\hline Tetraplegia & $40(13.1)$ \\
\hline Type of SCI Complete & $290(95.1)$ \\
\hline Incomplete & $15(4.9)$ \\
\hline \multicolumn{2}{|l|}{ American SCl Association Impairment Scale } \\
\hline$A$ & \\
\hline B & $290(95.1)$ \\
\hline $\mathrm{C}$ & $7(2.3)$ \\
\hline $\mathrm{D}$ & $6(2)$ \\
\hline & $2(0.7)$ \\
\hline
\end{tabular}


Table 2

Rotated Component Matrix ${ }^{a}$

Items

1. My health condition due to spinal

$\begin{array}{llllllll}1 & 2 & 3 & 4 & 5 & 6 & 7 & 8\end{array}$

.846 cord injury is stressful for me.

1. Following health problems caused .636 by my spinal cord injury, I often worry about my financial situation and living expenses.

1. People and my family do not see my ability as a healthy person.

1. I always think I need mobility aids

(wheelchair, cane-walker).

1. I suffered early due to health problems caused by spinal cord injury.

1. Because of the health problems caused by my spinal cord injury, I prefer to be alone with friends and acquaintances.

1. My health condition after spinal cord injury has made me resilient and strong in the face of life's problems. 2

1. I have the ability to deal with most problems caused by disability. 2

1. I use of all the remaining capacities for my growth and development. 2

1. The health problems caused by spinal cord injury do not prevent me from being interested in planning for the future. 2 
1. I have dealt with my disabling

health problems but I have not given up.2

1. The disabling and chronic health condition caused by spinal cord injury strengthened my remembrance of God as a source of comfort to me.2

1. I want to change my lifestyle according to my physical disability.2

1. Immobility from spinal cord injury has taken away my ability to live a normal life.3

1. This health accident caused me to lose the important things in my life. 3

1. After a spinal cord injury, I feel like .692 I have lost my purpose in life. 3

1. Because of the health problems .681 caused by spinal cord injury, I have fewer choices about the important decisions in my life. 3

1. Accepting the health status caused by the spinal cord injury gives me better control over my condition. 4

1. I try to help adapt to the injury by learning about my health status after a spinal cord injury. 4

1. I give people with spinal cord

injuries like me the motivation and hope to overcome the problems caused by disability. 4

1. I have made a serious decision to increase my abilities. 4 
1. Despite the health problems caused by spinal cord injury, I have a sense of independence in doing my personal and caring affairs.4

1. After a spinal cord injury, my spiritual beliefs helped me cope with my health problems. 4

1. After a spinal cord injury, the .676 normal things in life have become valuable to me.5

1. Spinal cord injury has allowed me to have a closer relationship with my family. 5

1. Instead of focusing on my health, I see the positive aspects of the situation.5

1. I am always eager to overcome .603 my health problems by following the advice of health care providers. 5

1. My past experiences can help me cope with my new health condition.5

1. I am afraid of end of life because of chronic and disabling health problems. 6

1. Everyone's role in the family diminishes after this physical disability incident. 6

1. I always worry about being rejected by others because of health problems caused by my spinal cord injury. 6

1. I cannot believe my physical disability.7

1. Whenever I have a health problem 
caused by a spinal cord injury, I express feelings such as anger and sadness.7

1. Since I have not accepted my

injury, I am waiting for my health

to return every day.7

1. The way society treats me now

bothers me more than the

inappropriate urban environment.8

1. I am not able to use all the available resources to solve my health problems. 8

\section{Extraction Method: Principal}

Component Analysis.

Rotation Method: Varimax with Kaiser Normalization.

a. Rotation converged in 9 iterations.

Table 3

Stability Coefficients and ICC of the CAHSSTSCI Subscales

\begin{tabular}{|llcl|}
\hline & Factors Cronbach's a & & \\
Factors & & ICC & P-value \\
& & & Test-retest \\
& & & \\
\hline Perception threat due to SCI & 0.94 & 0.89 & 0.0001 \\
\hline Resilience & 0.96 & 0.93 & 0.0001 \\
\hline Losing & 0.99 & 0.99 & 0.0001 \\
\hline Compatibility & 0.76 & 0.62 & 0.0001 \\
\hline Post-traumatic growth & 0.69 & 0.60 & 0.0001 \\
\hline Vague understanding of being & 0.99 & 0.99 & 0.0001 \\
\hline Disbelief and anger & 0.94 & 0.89 & 0.0001 \\
\hline Improper communication with environment & 0.96 & 0.93 & 0.0001 \\
\hline
\end{tabular}




\section{Supplementary Files}

This is a list of supplementary files associated with this preprint. Click to download.

- Appendix.docx 